\title{
Protective Role of microRNA-200a in Diabetic Retinopathy Through Downregulation of PDLIMI
}

\author{
Wencui Wan* \\ Yang Long (D* \\ Xuemin Jin \\ Qiuming Li \\ Weiwei Wan (D) \\ Hongzhuo Liu \\ Yu Zhu
}

Department of Ophthalmology, The First Affiliated Hospital of Zhengzhou

University, Zhengzhou, Henan, People's

Republic of China

*These authors contributed equally to this work
Correspondence: Yu Zhu

Email13673666718@I63.com
Background: Diabetic retinopathy (DR) is a most common microvascular complication and regarded as the leading cause of blindness in the working age population. The involvement of miR-200a in various disorders has become recognized, and the objective of this study was to identify the protective effect of miR-200a in the development of DR.

Methods: The contents of miR-200a and its potential target gene, PDZ and LIM domain protein 1 (PDLIM1), were detected in both in-vivo and in-vitro DR models. Retinal leakage and inflammatory factor concentrations were detected after vitreous injections of miR-200a/PDLIM1 vectors in mice. The cellular viability, apoptosis and cellular migration were investigated using trypan blue staining, flow cytometry and transwell assay with human retinal microvascular endothelial cells (HRMECs). Besides, the prediction and confirmation of miR-200a targeting PDLIM1 were conducted with bioinformation analyses and dual-luciferase reporter assay.

Results: Lower miR-200a and higher PDLIM1 levels were detected in both in-vivo and invitro DR models. Besides, it was found that miR-200a treatment would significantly inhibit retinal permeability and inflammatory factors. Through targeting PDLIM1, it was found that miR-200a could improve cellular viability, remit apoptotic status and reduce cellular migration significantly in high glucose-treated HRMECs.

Conclusion: Our results demonstrated that miR-200a could be used as a potential therapy target through down-regulating PDLIM1 in DR.

Keywords: microRNA 200a, diabetic retinopathy, PDZ and LIM domain protein 1, endothelial cells

\section{Introduction}

The incidence rate of diabetes mellitus (DM) keeps rising and it has been estimated that there are 366 million cases of DM worldwide. ${ }^{1}$ As the prevalence rate of diabetic retinopathy (DR) has increased among the patients with a diabetes duration over 20 years, the management of DR would cause huge medical and economic resource pressure. ${ }^{2}$ DR, which is a most common microvascular complication, has been regarded as the principal cause of blindness in the working age population. Vision loss in DR patients is associated with the development of proliferative diabetic retinopathy (PDR), including retinal neovascularization due to retinal ischemia and diabetic macular edema (DME) due to vascular leakage. The pathological characteristics of retinal tissue in DR patients include pericyte and endothelial cell (EC) loss, blood-retinal barrier (BRB) breakdown and retinal neovascularization. ${ }^{3}$ In the latest stage, relentless abnormal fibrovascular proliferation would appear and a vitrectomy would be conducted to rescue the retinal tissue and visual function. 
Endothelial dysfunction, which is also one of the main complications of DM, plays a key role in the development of various diabetic complications, including visual impairment, kidney failure, stroke, diabetic cardiomyopathy and lowerextremity dysfunction. ${ }^{4}$ During the development of DR, capillary nonperfusion would lead to increased proinflammatory factors because of retinal ischemia/hypoxia. Clear evidence from both DM animal models and clinical patients have demonstrated that inflammation plays an essential role in the pathogenesis of DR. ${ }^{5}$ As reported in a previous study, it has been found that pro-inflammatory growth factors and cytokines, such as tumor necrosis factor alpha (TNF $\alpha)$, interleukin 6 (IL6), interleukin $1 \beta$ (IL1 $\beta$ ) and monocyte chemo-attractant protein-1 (MCP-1), are overexpressed in clinical samples of patients with DR. ${ }^{6}$ Increased expression of inflammatory factors lead to BRB breakdown, and then damaged $\mathrm{BRB}$ is a key pathological progression in the incidence of macular edema. In a later stage of DR, angiogenesis would also be induced by the up-regulation of proangiogenic factors angiopoietin 2 (Ang 2) ${ }^{7}$ and vascular endothelial growth factor (VEGF). ${ }^{8}$ Abnormal expression of pro-angiogenic factors would lead to retinal neovascularization, which is the indicator of PDR. It is interesting to find that both Ang 2 and VEGF are important pro-inflammatory factors as well and provide important regulation effect in DR. ${ }^{7,9}$ Anti-VEGF therapy is a breakthrough in the management of DR, including its application in the treatment of DME and an adjuvant of vitrectomy. ${ }^{10,11}$ The progress in DM management and DR treatment has not provided guaranteed success in the treatment or prevention of progression of PDR. ${ }^{12}$ Considering the pivotal role of EC inflammation in the development of DR, advanced researches in the molecular mechanisms are still urgently required to identify potential therapy target.

MicroRNAs (miRNAs) are a class of non-coding RNA with 19-25 nucleotides, and they can regulate gene expressions through posttranscriptional repression of target gene expressions by binding to their $3^{\prime}$ untranslated regions ( 3 '-UTRs). It has been found that miRNAs expression profile was dysregulated in multiple retinal diseases, ${ }^{13,14}$ including DR. ${ }^{15,16}$ Recent studies suggested that the development and progression of DR could be regulated by several miRNAs, including miR-145-5p, miR-30a and miR-125b-5p/miR-146a-5p, through regulating retinal ganglion cell survival, retinal microglial activation and retinal microvascular injury, respectively. ${ }^{17-19}$ The miR-200 family is one of the best investigated functional miRNAs. It is composed of five highly conserved
miRNAs, including miR-141, miR-429 and miR-200a/ 200b/200c. A study by Ding et al showed that miR-200b/ c could protect vascular ECs under diabetic status by inhibiting their target gene, vasohibin-2. However, the effects of miR-200a in the development and progression of DR remains poorly understood. ${ }^{20}$

Considering the key pathological role of EC inflammation and important regulation potential of miR-200a in DR progression, it would provide us more knowledge to investigate miR-200a expression patterns during DR development and to identify the effects of miR-200a as a protective factor. As retinal EC dysfunction could lead to an increased vascular permeability at an early stage and angiogenesis at a late stage, the potential EC inflammation protector, miR-200a, would uncover the potential application of miR-200a as an effective agent for inhibition of DR progression.

\section{Methods and Materials Mouse DM Animal Model}

Male C57BL/6J mice (7-8 weeks old) were purchased from the Vital River Laboratory (Beijing, China), and their weights and glycemia levels were quantified and recorded. Streptozotocin (STZ, SigmaAldrich, USA) at $55 \mathrm{mg} / \mathrm{kg}$ in diluted $10 \mathrm{mM} / \mathrm{L}$ citrate buffer ( $\mathrm{pH} 4.5$ ) was injected intraperitoneally to the mice for 5 consecutive days. Age-matched male C57BL/6J mice were injected with citrate buffer and used as control in this study. The blood glycemia from the tail vein was measured a week after the last STZ injection. Mice were considered diabetic when three consecutive measurements of blood glucose were higher than $17 \mathrm{mM}(300 \mathrm{mg} / \mathrm{dL})$. The mice were maintained under standard conditions for lighting (a $12 \mathrm{~h} /$ $12 \mathrm{~h} \mathrm{light/dark}$ cycle), temperature $\left(23^{\circ} \mathrm{C}-25^{\circ} \mathrm{C}\right)$ and humidity $(50 \%-60 \%)$. The care and handling of animals were conducted in accordance with the Association for Research in Vision and Ophthalmology Statement for the Use of Animals in Ophthalmic and Vision Research. The animal protocol was approved by Zhengzhou University's Institutional Animal Care and Use Committee.

\section{Intravitreal Injection}

For the transfection of plasmid and miRNA mimics in animal models, in-vivo jetPEI transfection reagent according to the manufacturer's instructions was used in this study. The mice were positioned in lateral recumbency, eyes were protruded by applying subtle pressure around 
the eyeballs, and the needle was injected through the sclera into the vitreous. A total volume of $1 \mu \mathrm{L}$ liquid was used for each intravitreal injection, and an injection posterior of the limbus was conducted using a 33-gauge needle (Hamilton, USA) without damaging the lens under an operating microscope (Carl Zeiss Surgical, NY, USA). For plasmid in-vivo transfection, $1.0 \mu \mathrm{L}$ plasmid in $4.0 \mathrm{mg} / \mathrm{mL}$ was used in the injection. For miRNA injection, miR-200a mimic or miRNA normal control (miR$\mathrm{NC}$ ) with a concentration of $4.0 \mathrm{mg} / \mathrm{mL}$ was used in the invivo transfection. The mice were randomly divided into 6 groups, and the mice in each group received an intravitreal injection of $1 \mu \mathrm{L}$ phosphate buffer saline (PBS) in normal mice (control group), $1 \mu \mathrm{L}$ PBS in diabetic mice (DM group), $1 \mu \mathrm{L}$ of PDLIM1 expression plasmid in diabetic mice (DM+PDLIM1 group), $1 \mu \mathrm{L}$ of miR-200a in diabetic mice (DM+miR-200a group), $1 \mu \mathrm{L}$ of PDLIM1 expression plasmid combined with miR-200a in diabetic mice (DM +PDLIM1+miR-200a group) and $1 \mu \mathrm{L}$ of PDLIM1 expression plasmid combined with miR-NC in diabetic mice (DM+PDLIM1+miR-NC group). All the following experiments were conducted three days after injection.

\section{Retinal Thickness Measurement}

The retinal cross-sectional thicknesses were detected using a spectral domain optical coherence tomography (SDOCT) system (Heidelberg Engineering, Heidelberg, Germany) in this study. In general, animals in different groups were anesthetized with $100 \mathrm{mg} / \mathrm{kg}$ ketamine and $12 \mathrm{mg} / \mathrm{kg}$ xylazine mixture, and the OCT images were simultaneously captured on retinal locus at $30^{\circ}$ from the optic nerve head. The total retinal thickness was defined as the value from retinal pigment epithelium to the photoreceptor outer segments at $500 \mu \mathrm{m}$ from the optic disc in nasal-temporal sectors. A total of three images for each eye were used in the measurement of retinal thickness, and the mean value was used in this study.

\section{Enzyme-Linked Immunosorbent Assay for Inflammatory Factors}

The neuro-retina tissues were extracted from eyes of mice in different groups. After extracting the protein in lysis buffer containing protease inhibitor, the sonicated suspension was centrifuged at $2 \times 10^{5} \mathrm{~g}$ for $20 \mathrm{mins}$ at $4^{\circ} \mathrm{C}$. The supernatant from all the retinal extracts was used to measure the protein levels of IL-1 $\beta$, TNF $\alpha$, IL6 and ICAM1 with enzyme-linked immunosorbent assay (ELISA) kits purchased from Research \& Diagnostics Systems (IL-1 $\beta$, MLB00C; TNFa, MTA00B; IL6, M6000B and ICAM1, DY796). The levels of these inflammatory factors in mouse retinal samples were determined according to the protocols provided by the manufacturers. The samples were applied to the ELISA kits after validation, and the optical density was determined at 450 and $540 \mathrm{~nm}$ with a microplate reader (FLUOstar Omega-Microplate reader, Germany).

\section{Retinal Vascular Leakage Assay}

Retinal leakage assay was conducted based on literature with some modifications in previous studies. ${ }^{21}$ In different mouse groups, including control, DM, DM +PDLIM1, DM+miR-200a, DM+PDLIM1+miR-200a and $\mathrm{DM}+\mathrm{PDLIM} 1+\mathrm{miR}-\mathrm{NC}$ groups, $45 \mathrm{mg} / \mathrm{kg}$ Evans Blue (Sigma-Aldrich Corp. St. Louis, MO, USA) in PBS was injected through a jugular vein catheter. After 1-hour circulation, heart perfusion via the left ventricle was conducted with PBS followed by $1 \%$ paraformaldehyde, and the mice were sacrificed by $\mathrm{CO}_{2}$ overdose. The retinal samples were freshly dissected for qualitative measurement and the Evans Blue dye in each retinal sample was extracted by incubating in $50 \mu \mathrm{L}$ formamide (Sigma, USA) overnight at $70^{\circ} \mathrm{C}$. The extracted Evans Blue was measured at an absorbance at 620 $\mathrm{nm}$ after 70,000 $\mathrm{g}$ ultracentrifugation for $1 \mathrm{~h}$. The ratio of the leakage intensity compared to the intensity of control group was used in this study.

\section{Cell Culture}

Human retinal microvascular endothelial cells (HRMECs) were from American Type Culture Collection (ATCC, Manassas, VA), and HRMECs at 4 to 8 passages were used in this experiment. The cells were cultured in precoated flasks with gelatin (Corning, NY, USA). The cells were cultured in endothelial basal medium (Lonza; Walkersville, MD, USA) supplemented with $10 \%$ fetal bovine serum (FBS), $100 \mathrm{U} / \mathrm{mL}$ penicillin and $100 \mu \mathrm{g}$ / $\mathrm{mL}$ streptomycin and EC growth supplements (Lonza; Walkersville, MD, USA). The $293 \mathrm{~T}$ cells were cultured in DuIbecco's modified eagIe's medium (DMEM) with $10 \% \mathrm{FBS}, 100 \mathrm{U} / \mathrm{mL}$ penicillin and $100 \mu \mathrm{g} / \mathrm{mL}$ streptomycin. The cells were cultured at $37^{\circ} \mathrm{C}$ and $5 \%$ vol $\mathrm{CO}_{2}$, and the culture medium was replaced once every three days.

\section{Cell Treatment}

An effective PDLIM1 plasmid was designed and synthesized by Beijing Zhongyuan Company. The miR-200a 
overexpression mimics $\left(5^{\prime}-\right.$

UAACACUGUCUGGUAACGAUGU - $3^{\prime}$ ) and the nonsense miRNA (miR-NC) as a negative control (5'UUCUCCGAACGUGUCACGUTT-3') were synthesized by GenePharma company (Shanghai, China). The negative control miR-NC, miR-200a mimic and PDLIM1 plasmid were transfected into the cultured cell using Lipofectamine 2000 (Thermo Fisher Scientific, USA), and all the operating procedures were conducted according to the manufacturer's instructions. The $80 \%$ confluent cells were starved in a minimal medium (basic medium $+2 \%$ FBS) overnight before transfection to eliminate the influencing effects of growth factors. Transfected cells were used for advanced experimental analysis at $48 \mathrm{~h}$ post-transfection.

\section{RNA Extraction and Quantitative PCR Assay}

Total RNA from both mouse retinal tissues and HRMECs was extracted with Trizol $^{\circledR}$ Reagent (Thermo Fisher Scientific, MA, USA) and purified using RNeasy Kit (Qiagen, USA) according to the instruction manual. Purified RNA was quantified at A260/A280 ratio was conducted with NanoDrop Spectrophotometers (ThermoFisher Scientific, MA, USA), then $500 \mathrm{ng}$ total RNA was used for cDNA synthesis with reverse transcription into cDNA using High Fidelity 1st Strand cDNA Synthesis Kit (Agilent Technologies, CA, USA), according to the manufacturer's instruction. Real-time qPCR was conducted by using an Applied Biosystem CFX1000 (Thermo Fisher Scientific, USA) and a SYBR Premix Ex Taq (Takara), according to the manufacturer's instructions. Each sample was measured in triplicate, and the mean value was used in data analyses. Each treatment was determined using five to six eyes, and data are shown as means $\pm \mathrm{SE}$. All the gene expressions were normalized to housekeeping gene glyceraldehyde-3-phosphate dehydrogenase (GAPDH) or U6 small nuclear RNA. The PCR reactions were performed in triplicate, and the data were analyzed by $2^{-\Delta \Delta \mathrm{Ct}}$ method.

The sequences of primers were as follows:

PDLIM1: forward 5'- GAGAAACAGGAGTTG AATGAGCC-3',

reverse 5'- GCAGCCACTTTAGTGACAGGAG-3'. miR-200a: forward 5'- CTTACCGGACAGTGCTG-3', reverse 5'- GAACATGTCTGCGTATCTC-3'.

GAPDH: forward 5'-GAAGGTGAAGGTCG GAGTC-3',

reverse 5'-GAAGATGGTGATGGGATTTC-3'.

\section{Cellular Viability Assay}

The cellular viability was detected with 3-(4,5-dimethylthiazol-2-yl)-2,5-diphenyltetrazolium bromide (MTT) assay kit following the manufacturer's instruction (\#30006, Biotium, USA). Cultured HRMECs $\left(5 \times 10^{4}\right.$ cells $\left./ \mathrm{mL}\right)$ in different groups were plated in a 96well plate 48 hours after the treatment, and $10 \mu \mathrm{L}$ CCK- 8 solution was added to each well. After incubation for 2 $\mathrm{h}$ at $37^{\circ} \mathrm{C}$, the absorbance values were measured by a multiplate reader (Lambda Bio-20; Beckman) at $450 \mathrm{~nm}$.

\section{Flow Cytometry for Apoptosis Analyses}

In this study, flow cytometry with annexin V fluorescein isothiocyanate (FITC) and propidium iodide (PI) stains was used to detect the apoptosis with FITC Annexin V Apoptosis Detection Kit I according to the manufacturer's instruction (BD, Biosciences, USA). After digestion with $0.25 \%$ trypsin and centrifuging at $400 \mathrm{~g}$ for 5 min, the HRMECs in all the groups were resuspended in ice-cold PBS and used for analyses. The annexin V-FITC and PI stains were added in the resuspension and incubated in the dark for $20 \mathrm{~min}$. Then the HRMEC suspension was passed through a $250-\mu \mathrm{m}$ mesh to prevent clumping before fluorescence analyses and data analyses. The fluorescence emission was detected at a $\lambda \mathrm{Em}$ of 518 and 620 $\mathrm{nm}$ for annexin V-FITC and PI, respectively, with FACS LSR II ${ }^{\circledR}$ (BD Biosciences). Data were analyzed using FlowJo $^{\circledR}$ VX software (Tree Star, OR, USA). The ratio of the percentage of the alive cell rate compared to that of the control group was calculated and used in data analyses.

\section{Cellular Migration Assay}

The transwell assay was used in the detection of migration ability of the HRMECs in different groups with Costar Transwell plates (Corning, MA, USA). Transwell migration assays were conducted and analyzed as in previous study. ${ }^{22}$ After seeding the HRMECs in different groups to the upper chambers and placing conditioned medium in the lower chambers for 24 hours, crystal violet was used to stain the cells. The migrated and stained cells were observed under an optical microscope, and six different fields were chosen at random for each sample. The mean value of each group was used for data analyses.

\section{Western Blot}

After treatments in different groups, HRMECs were lysed with Radio Immunoprecipitation Assay (RIPA) buffer 
(Biyuntian, Shanghai, China). Lysates were centrifuged at $14,000 \mathrm{~g}$ for $20 \mathrm{~min}$, and the supernatant was used for protein separation by dodecyl sulfate, sodium saltpolyacrylamide gel electrophoresis (SDS-PAGE). A total $30-50 \mu \mathrm{g}$ protein of total protein was separated by electrophoresis through 10-12\% SDS-polyacrylamide gel. Rabbit anti-Bax, anti-Bcl2 anti-cleaved caspase3, antiPDLIM1 and anti-GAPDH monoclonal antibodies (Cell Signaling Technology, USA) were used to detect total protein levels of Bax, Bcl2, cleaved caspase3, PDLIM1 and GAPDH. All antibodies were used at 1:1000 dilution. The Western blot bands were quantified using a luminescent image analyzer, ImageQuant LAS 4000 (GE Healthcare). The optical density of the target genes was normalized to GADPH.

\section{Dual-Luciferase Reporter Assay}

The target genes of miR-200a were predicted by TargetScan and miRcode. After selecting an overlapped gene, PDLIM1, the potential inhibition of miR-200a on PDLIM1 expression was confirmed by dual-luciferase reporter assay. The 3-UTR sequence of PDLIM1 was amplified by PCR from normal human genomic DNA database and subcloned into downstream of the stop codon pGL3-basic vector. The QuikChange SiteDirected Mutagenesis Kit was applied to mutate the binding site of miR-200a in PDLIM1. Next, the 293T cells $\left(5 \times 10^{4}\right)$ were seeded into 24 -well plates and cotransfected with wild-type or mutant 3-UTR vectors in combination with miR-200a or miR-NC for $48 \mathrm{~h}$. The regulation effect of miR-200a on the PDLIM1 expression was demonstrated by the Firefly luciferase activity and was normalized to Renilla luciferase activity and in both miR-200a and miR-NC groups. We used a dual-luciferase reporter system (Promega Corporation, Madison, WI, USA) to detect the luciferase activity according to the manufacturer's instructions. All experiments were repeated at least three times, and the average value was used in the data analyses.

\section{Statistical Analyses}

Statistical analyses and graph development were performed using GraphPad Prism 7 (GraphPad Software, USA). Statistical significance was determined using the $t$-test or one-way ANOVA. A $P$-value of less than 0.05 was regarded as a statistical significance.

\section{Results}

\section{Dynamic Expressions of miR-200a and PDLIMI in Both in-vivo and in-vitro DR Model}

The levels of miR-200a and PDLIM1 were analyzed by both in-vivo and in-vitro DR model at different time points. For in-vivo animal models, the miR-200a was up-regulated at both 2 months and 4 months after STZ injection, while both mRNA and protein levels of PDLIM1 were significantly increased at 2 months and 4 months DM stage (Figure 1A-C). On the other hand, we also detected the miR-200a and PDLIM1 expression at $12 \mathrm{~h}, 24 \mathrm{~h}$ and 48 $\mathrm{h}$ after high-glucose $(\mathrm{HG})$ treatment. For miR-200a, it was found that it was down-regulated in HRMECs treated with HG after $24 \mathrm{~h}$ and $48 \mathrm{~h}$, respectively (Figure 1D). The results of the mRNA and protein expressions of PDLIM1 at $12 \mathrm{~h}, 24 \mathrm{~h}$ and $48 \mathrm{~h}$ showed that they were significantly higher after $48 \mathrm{~h}$ and $24 \mathrm{~h}$, respectively, comparing with control group $(P<0.001$, Figure $1 \mathrm{E}$ and $\mathrm{F})$. However, no significant differences were detected in the PDLIM1 mRNA between $12 \mathrm{~h}$ or $24 \mathrm{~h}$ group with and control group $(P>0.05)$.

\section{miR-200a Eliminated Retinal Permeability Induced by Diabetes and PDLIMI}

As retinal permeability was one of the key signs for DR, both retinal thickness and retinal leakage were used. All the mice were divided into six groups, including control, DM, DM+PDLIM1, DM+miR-200a, DM+PDLIM1 group + miR-200a and DM+PDLIM1 group+miR-NC group. The retinal thickness in DM mice was not significantly different comparing with the age- and gender-matched normal mice; however, the retinal thickness value increased after treatment with miR-200a/miR-NC in combination with PDLIM1 vector. The representative OCT images in all the 6 groups are presented in Figure 2A, and the data are presented in Figure 2B. Comparing the DM mice, PDLIM1 treatment significantly increased the retinal thickness, and this effect was improved by miR-200a injection. In the diabetic mice, it was found that the retinal leakage was significantly increased compared with the normal group. Comparing with diabetic mice without any treatments, it was demonstrated that miR-200a and PDLIM1 produced protective and harmful effects, respectively. To evaluate the possible beneficial effect of miR200a on retinal leakage in DR, normal and STZ-induced 


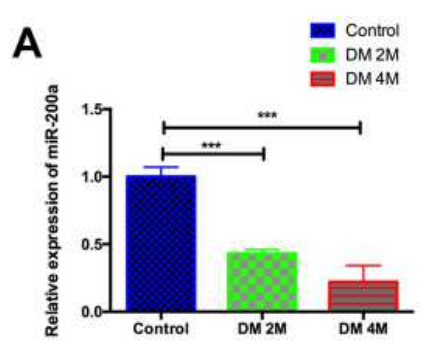

D

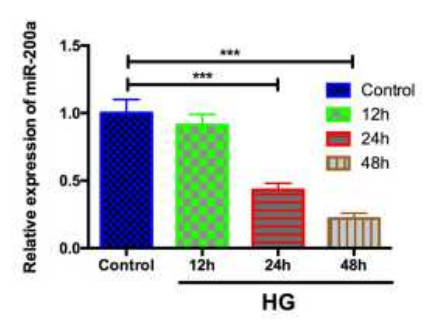

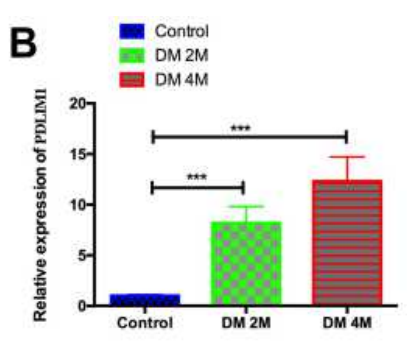

E

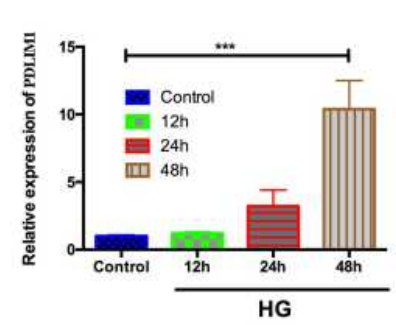

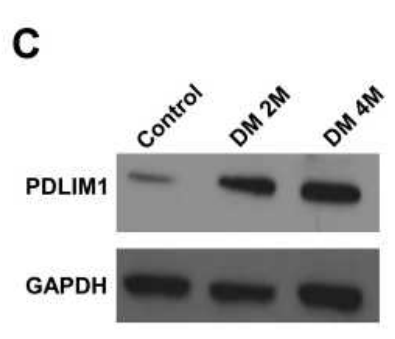

$\mathbf{F}$

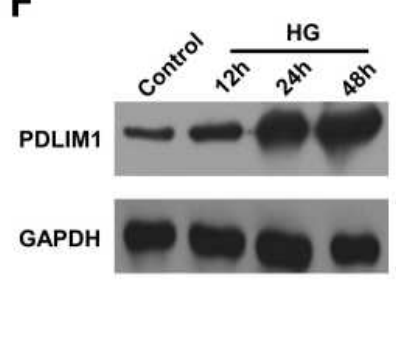

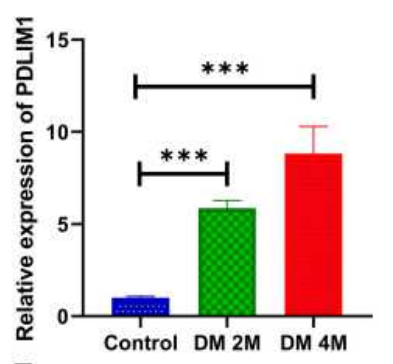

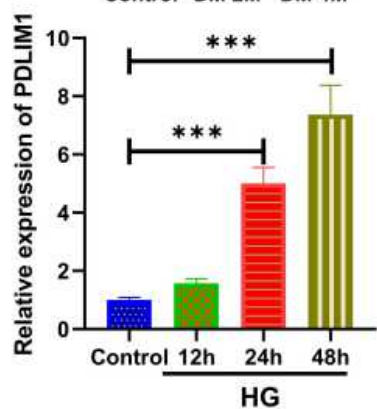

Figure I Expressions of miR-200a and PDLIMI at different time points in both in-vivo and in-vitro models. (A) miR-200a expression was detected in normal C57BL/6] mice, 2 months and 4 months after STZ injection. The expression of miR-200a was significantly decreased at 2 months and 4 months after STZ injection ( $n=6$ ); (B) PDLIMI mRNA expression was detected in normal C57BL/6] mice, 2 months and 4 months after STZ injection. The expression of PDLIMI mRNA was significantly increased at 2 months and 4 months after STZ injection ( $n=5)$; (C) PDLIMI protein levels in normal C57BL/6 J mice, 2 months and 4 months after STZ injection. The expression of PDLIMI mRNA was significantly increased at 2 months and 4 months after STZ injection $(n=4)$. (D) miR-200a expression was detected in HRMECs under normal glucose (NG), and I 2 h, 24 $\mathrm{h}$ and $48 \mathrm{~h}$ after high glucose (HG) treatment. The expression of miR-200a was significantly decreased at $24 \mathrm{~h}$ and $48 \mathrm{~h}$ after HG treatment; (E) PDLIMI mRNA expressions were detected in HRMECs under NG, and $12 \mathrm{~h}, 24 \mathrm{~h}$ and $48 \mathrm{~h}$ after HG treatment. (F) PDLIMI protein levels were detected in HRMECs under NG, and $12 \mathrm{~h}, 24 \mathrm{~h}$ and 48 $\mathrm{h}$ after HG treatment $(n=4)$. (Data are expressed as mean \pm SD, $* * * P<0.001)$.

diabetic mice were treated with miR-200a/miR-NC in combination with PDLIM1, and then retinal vascular leakage was investigated using Evans Blue assay. The in-vivo findings demonstrate that retinal microvascular leakage increased in the diabetes group. After intravitreal injection of PDLIM1 plasmid, it was found that PDLIM1 could induce retinal leakage in diabetic mice. To directly test the effects of miR-200a in retinal microvascular leakage, an miR-200a mimic/miR-NC mimic in combination with PDLIM1 was intravitreally injected to diabetic mice, and the leakage index was detected two days later (Figure 2C). These results indicated that intravitreal injection of miR200a could be an efficient therapy method for both diabetic mice and PDLIM1-induced inflammatory leakage invivo.

\section{Effect of miR-200a on Inflammatory Cytokine Levels in the Retinal Samples of Diabetic Mice}

Given that inflammation plays an important role in the development of DR and it is a key factor for increased retinal permeability, it is important to detect the inflammatory factor expression in different groups. As miR-200a was a potential inflammation inhibitor that protected diabetic retinal development, we hypothesized that miR200a might influence EC permeability through interaction with inflammatory molecules such as IL-1 $\beta$, TNF $\alpha$, IL6 and ICAM1 (Figure 3). The expressions of the four inflammatory factors were examined in the retinal tissues from different groups, and the effects of miR-200a on PDLM1 treatment were demonstrated. Comparing with the ageand gender-matched control mice, the expressions of IL$1 \beta$, IL6 and ICAM1 were significantly increased, and ICAM1 demonstrated the most significant increase. After treatment with PDLIM1, the expressions of all the four inflammatory factors were significantly up-regulated. After treatment with miR-200a, the levels of IL-1 $\beta$, IL6 and ICAM1 in untreated diabetic mice were decreased at 12 $\mathrm{h}$, while the TNF $\alpha$ level was still higher than the untreated diabetic mice group at $48 \mathrm{~h}$.

\section{miR-200a Relieved HRMECs Viability, Apoptosis and Migration Influenced by HG}

To further address how miR-200a modulated HRMEC function in $\mathrm{HG}$ status, we detected the effects of miR200a on cell viability of both normal and high-glucose treated HRMECs (Figure 4). For the HRMECs in normal 
A

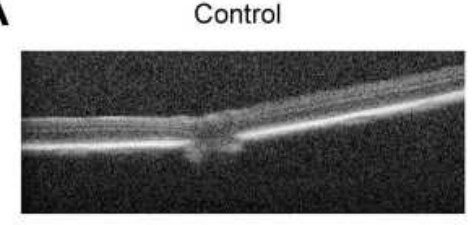

$D M+m i R-200$

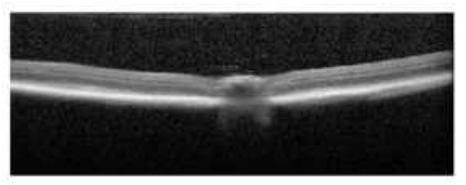

B

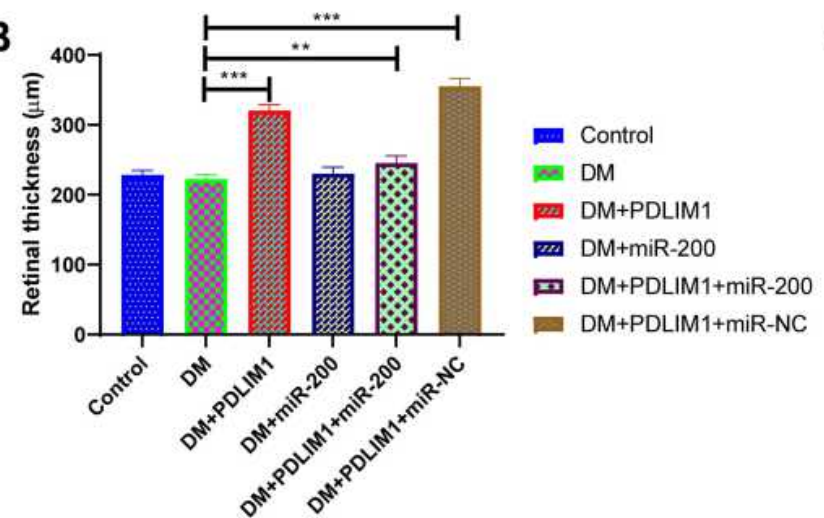

DM

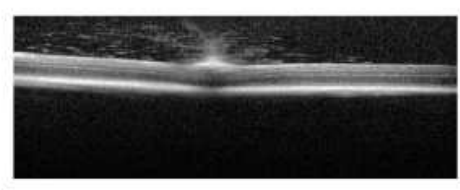

DM+PDLIM1+miR-200
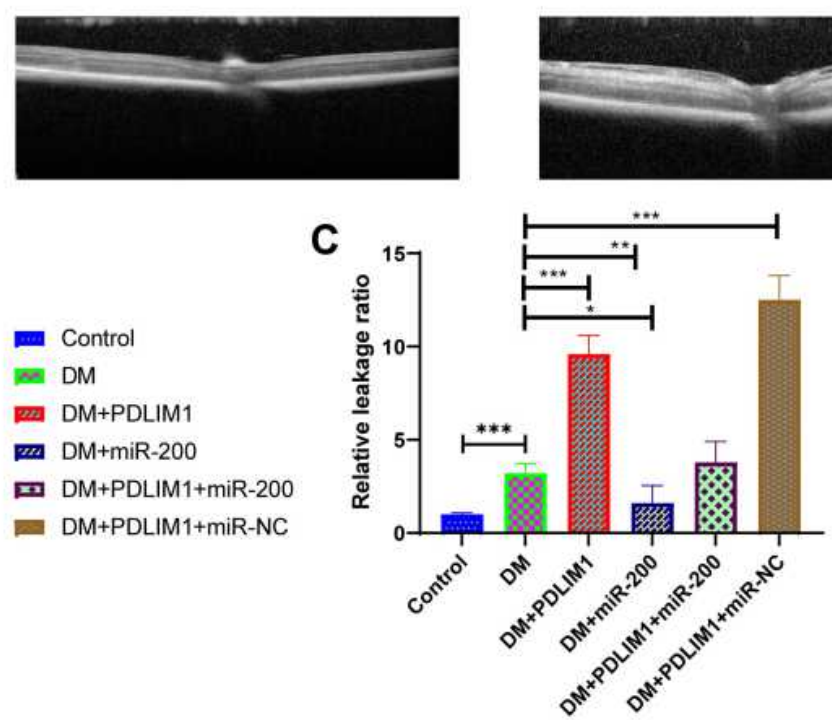

DM+PDLIM1

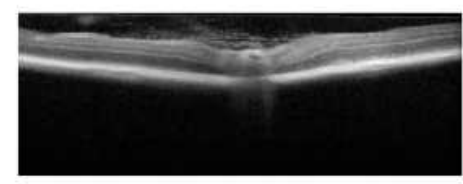

$\mathrm{DM}+\mathrm{PDLIM} 1+\mathrm{miR}-\mathrm{NC}$

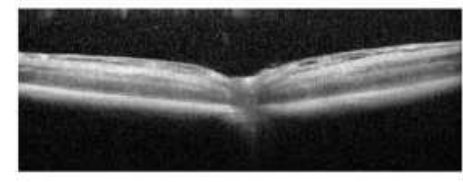

m Control

DM

DM+PDLIM1

m DM+miR-200

Ш DM+PDLIM1+miR-200

- DM+PDLIM1+miR-NC

Figure 2 Effects of miR-200a, PDLIMI and diabetic status on retinal thickness and leakage. (A) Optical coherence tomography (OCT) images of retinas for different groups, including normal, DM, DM+PDLIMI, DM+miR-200a, DM+PDLIMI+miR-200a, DM+PDLIMI+miR-NC; (B) retinal thickness based on OCT in normal, DM, DM+PDLIMI, DM+miR-200a, DM+PDLIMI+miR-200a, DM+PDLIMI+miR-NC groups; (C) retinal leakage based on Evans Blue assay in normal, DM, DM+PDLIMI, DM+miR-200a, DM $+P D L I M I+m i R-200$ a, DM+PDLIMI+miR-NC group. Data are expressed as mean $\pm S D$. $* P<0.05, * * P<0.01, * * * P<0.001$.

glucose, miR-200a treatment had no effect on the cell viability. After treatment with $\mathrm{HG}$ for $24 \mathrm{~h}$, it was found that cell viability was significantly influenced, however; the application of miR-200a improved the HRMECs viability significantly $(P<0.001)$.

Since apoptotic HRMECs are frequent under HG conditions, we determined whether miR-200a could relive HRMECs form of cell death. To detect the apoptotic rates in different groups, annexin $\mathrm{V}$ signal by using an anti-annexin $\mathrm{V}$ antibody and the expression of a group of apoptosis related protein, including Bcl2, Bax and cleaved caspase 3. As shown in Figure 5A, the percentage of apoptotic cells was increased from $5.1 \%$ in the control group to $11.6 \%$ in the HG group for HRMECs. After treatment with miR-200a for $24 \mathrm{~h}$, it was found that the percentage of apoptotic cells was reduced to $6.1 \%$, and it demonstrated a significant difference from that in HRMECs in the HG group. The results indicated that miR-200a treatment significantly protects the HRMECs under HG stress. In advanced studies, we detect the apoptosis-related protein expression (Figure 5B). Comparing with the control group, an increased expression of proapoptotic protein Bax and the apoptotic executioner cleaved caspase 3 was detected HRMECs in HG status. In advance, there was a decreased expression of antiapoptotic protein $\mathrm{Bcl} 2$, and there was a significant difference between $\mathrm{HG}$ and NG groups. After treatment with miR-200a in HRMECs under high glucose status, we found a significantly decreased pro-apoptotic protein Bax and cleaved caspase 3 expression and an increased antiapoptotic protein Bcl2 expression. However, no significant difference in the apoptosis-related proteins expression was observed for miR-NC treated HRMECs compared with HG treated group. Taken together, these results suggest that miR-200a protected HRMECs apoptosis by inhibiting the mitochondrial apoptosis pathway.

Comparing with the control group, HG led to an increased migration rate through transwell assay. Besides, miR-200a alleviated the HG-induced increasing cell migration, while miR-NC failed to demonstrate a regulatory effect (Figure 6A). Besides, the effects of HG, HG+miR-200a as well as HG+miR-NC are presented in Figure 6B. 

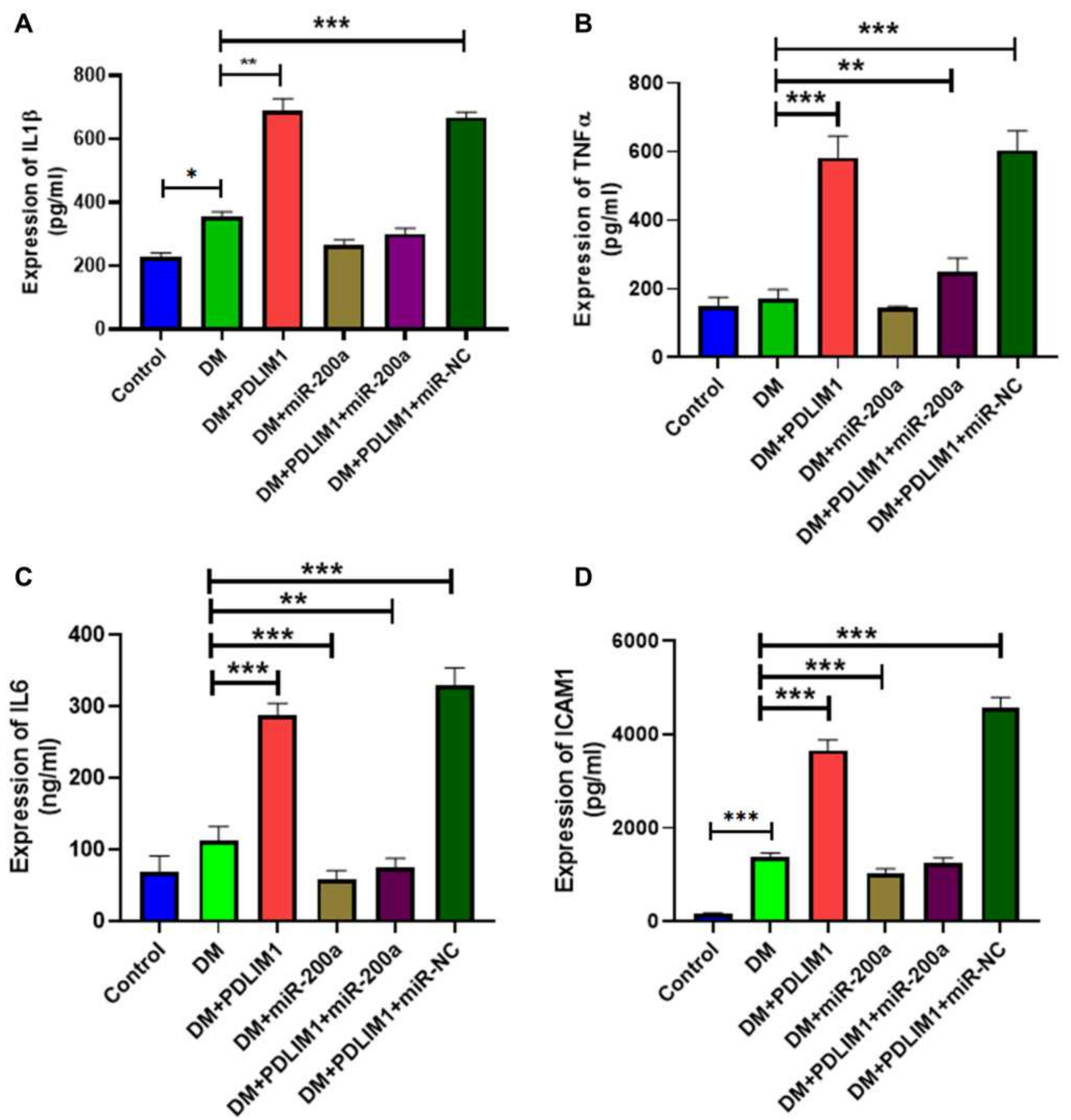

D

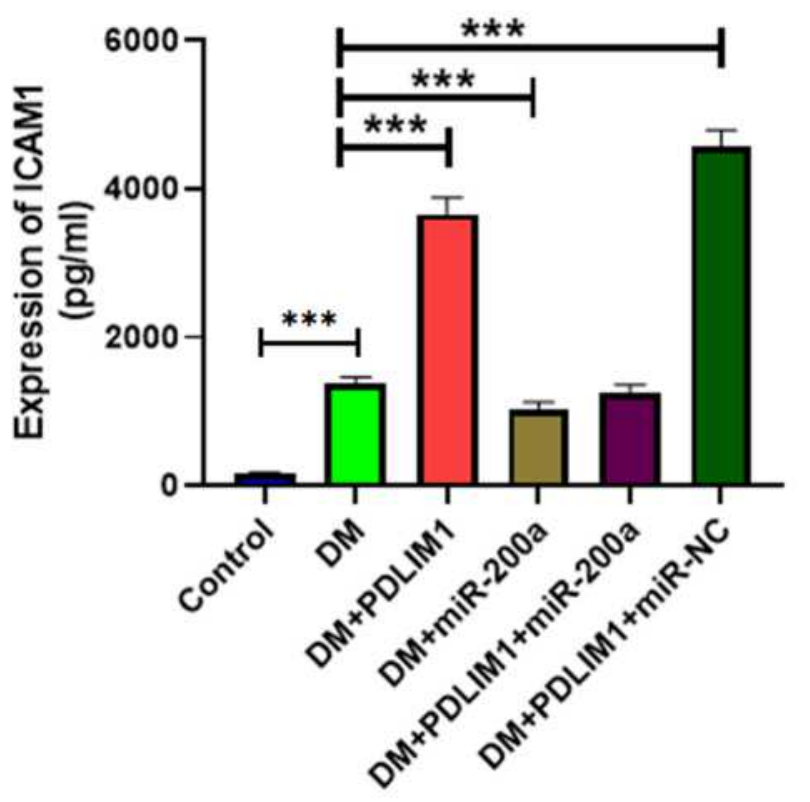

Figure 3 Effects of miR-200a, PDLIMI and diabetic status on pro-inflammatory factors. The expression of several pro-inflammatory factors was detected by ELISA and presented in (A) IL-I $\beta$, (B) TNF $\alpha$, (C) IL6 and (D) ICAMI. The pro-inflammatory was significantly induced by PDLIMI treatment and reduced by miR-200a. $* P<0.05$, $* * P<0.01, * * * P<0.001 . n=5$.

miR-200a Regulated HRMECs Function by Targeting PDLIMI

As we know, miRNAs have demonstrated a biological function through post-translational regulation. In this study, bioinformatics analysis was employed to predict the potential target genes of miR-200a by TargetScan and
miRcode. As PDLIM1 was significantly up-regulated in HG-treated HRMECs and its expression was remarkably reduced by treatment with miR-200a (Figure 7A and B), we conducted advanced studies on the regulation effect of miR-200a on PDLIM1 expression. After co-incubating miR-200a or miR-NC mimics with luciferase reporter 


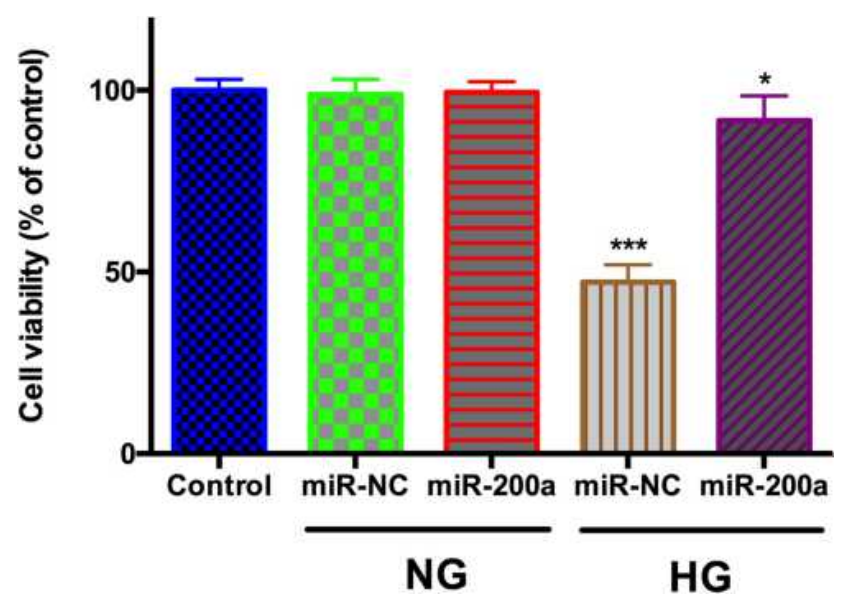

Figure 4 The cellular viability in normal glucose (NG) and high glucose (HG) group after treatment with miR-200a was assessed. miR-200a improved cellular viability in HG group. $* P<0.05, * * * P<0.001 . n=5$.

vector containing the wild-type or mutant $3^{\prime}$ UTR of the sequence, a significantly decreased luciferase activity was observed in the miR-200a and wild PDLIM1 cotransfection group (Figure 7C). The dual-luciferase reporter assay provided us the knowledge that PDLIM1 was a target gene of miR-200a.

\section{Discussion}

DR, which is a most important microvascular diabetesinduced complication, is regarded as a leading cause of vision disability. However, more efforts are still urgently required to understand the pathological mechanisms and develop novel approaches for the therapy. As the miR-200 family plays a key role in different biological progresses, here we demonstrated the potential protective effects of miR-200a for DR based on both in-vivo and in-vitro models. Through analyzing the retinal permeability and inflammatory biomarkers in streptozotocin diabetic mice after treatment with miR-200a and PDLIM1, we demonstrated the essential contribution of PDLIM1 in the development of DR, and miR-200a would provide remarkable protective effect through inhibiting the expression of PDLIM1. The results from in-vitro studies showed that treating HRMECs in HG with miR-200a would improve the cell viability as well as apoptotic status. Dual-luciferase reporter assay confirmed that miR-200a could demonstrate the biological through directly regulating PDLIM1 (Figure 8).

Among all the diabetic patients, about one-third of them suffered from vision-threatening DR, and DME was one of the most important causes of vision impairment. ${ }^{16}$ Focal laser photocoagulation is regarded as a classical treatment for DME; however, it leads to a number of serious complications. ${ }^{23}$ Data from several clinical trials demonstrated significant beneficial effects of anti-VEGF therapy in the management of DME, while anti-VEGF resistance was associated with poor prognosis of DME management. $^{24,25}$ Although a variety of scientific advances have been made over the last decade, the pathogenesis of DME remains to be discovered fully. By now the key role of inflammation in the development from early phases DR to vision-threatening DME has been reported by different researches and reviews, and the modification of retinal inflammation is regarded as a therapy target for DR. ${ }^{26-28}$ Mounting evidence has demonstrated the role miRNAs in the development of different disorders, ${ }^{29}$ and among them the miR-200 family is one of the most commonly studied. A previous study detected the protective effects of miR$200 \mathrm{~b} / \mathrm{c}$ through inhibiting vasohibin-2 in HRMECs. ${ }^{20}$ Another study was conducted to detect the effect of miR200c in Ishikawa cells and found that up-regulation of miR-200c could repress both transcription and translation of zinc finger E-box-binding proteins (ZEBs), VEGFA, fms-related tyrosine kinase 1 (FLT1), inhibitor kappa B kinase $\beta$ (IKK $\beta)$, as well as Krüppel-like transcription factor 9 (KLF9) through interacting with their 3'untranslated regions and then demonstrated significant biological effects. ${ }^{30}$ It was also reported that another member in the miR-200 family, miR-200a, could produce key regulation effects in diabetes-related diseases. In a study on other microvascular complications, such as diabetic nephropathy, it was found that a novel analogue of curcumin C66 could ameliorate diabetic nephropathy by up-regulating miR-200a. ${ }^{31}$ In a study on zebrafish, miR-200a was reported to be one of the most important functional miRNAs in retinal degeneration and it is essential in the Müller glia cellular reprogramming. ${ }^{32}$ However, no previous work has been conducted on the effects of miR-200a in the development of DR.

In this study, we detected the roles of miR-200a in the regulation of HRMECs inflammation, which was one of the key progresses in the development of DR, and the results in this study supported our hypothesis of miR200a having a protective effect in the development of DR. PDLIM1, also known as Coactosin-like protein 36 (CLP36), Elfin and Cofactors of LIM homeodomain protein 1 (CLIM1), contains a N-terminal PDZ domain and a C-terminal LIM motif. ${ }^{33}$ It was reported that PDLIM1 was related with subcellular location regulation of signal proteins and it also functioned as a stress fiber-associated 


\section{A}
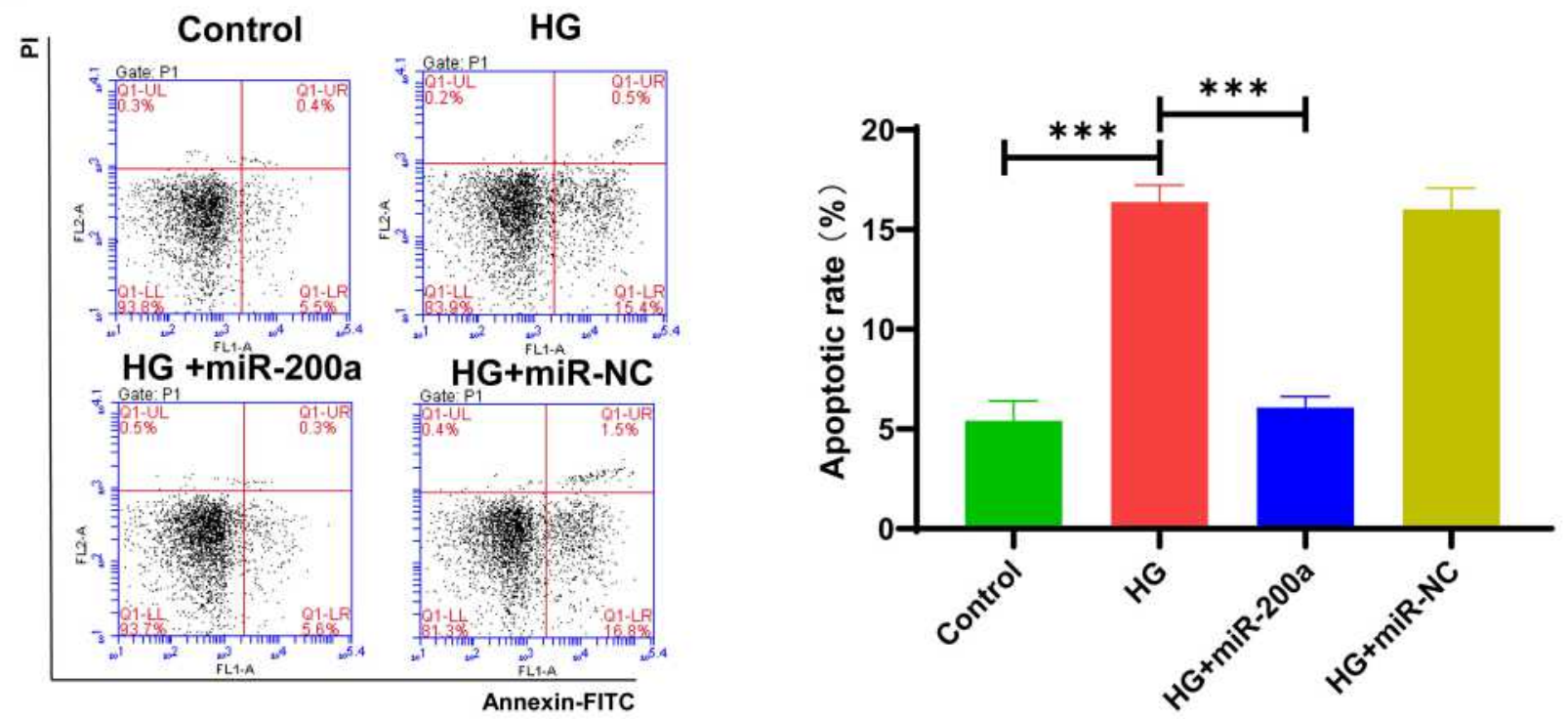

B
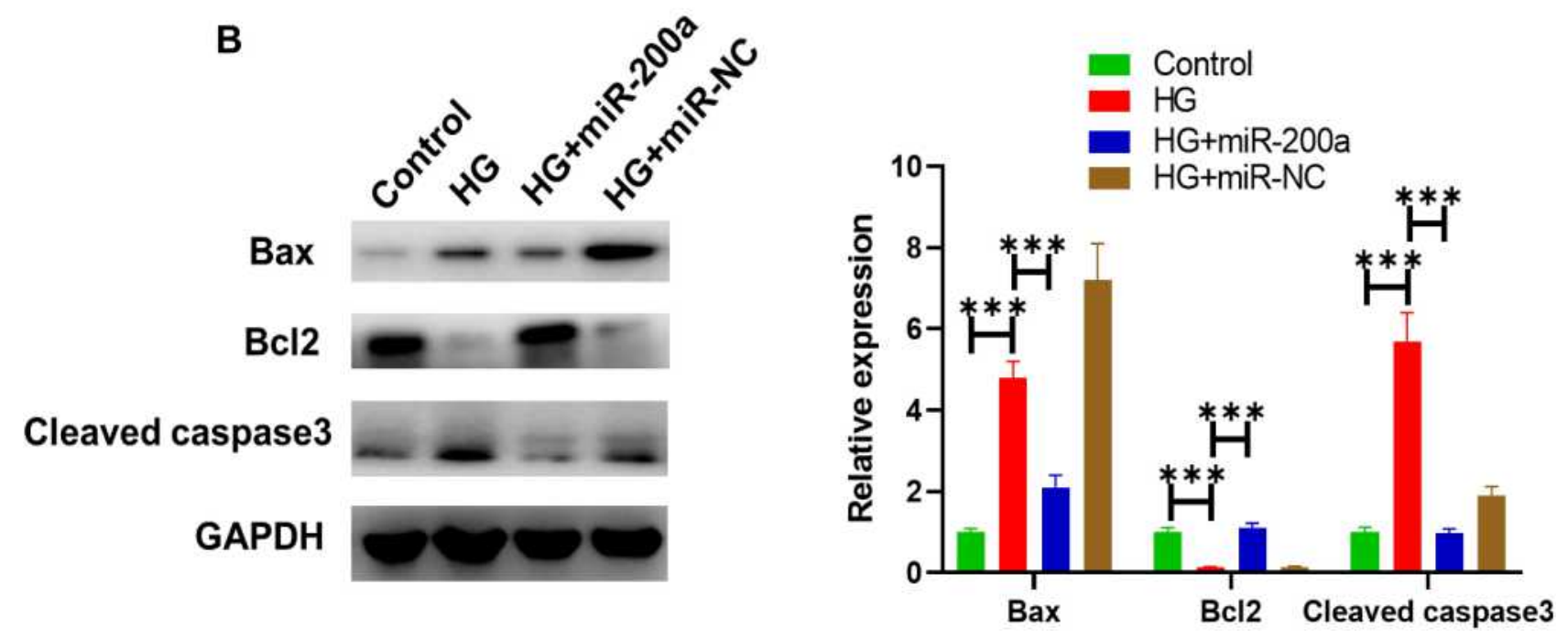

Figure 5 Effect of miR-200a on apoptosis and apoptosis-related genes in high glucose (HG)-treated HRMECs. (A) The apoptosis rate was detected in cultured HRMECs in normal glucose (NG), high glucose (HG), HG+miR-200a and HG+miR-NC. Summary of apoptotic rates in different groups is provided. HG treatment significantly increased apoptotic rate, while the apoptosis was improved by miR-200a treatment. $n=5$. (B) Role of miR-200a on the expression of apoptosis-related genes, including Bax, Bcl-2 and caspase-3, in HG-treated HRMECs by Western blotting. miR-200a treatment leads to pro-apoptosis gene down-regulation and anti-apoptosis gene up-regulation. $* * * P<0.001$.

cytoskeletal protein. However, the role of PDLIM1 in inflammation or ECs remained largely undefined and controversial. ${ }^{34,35}$ A recent study on the role of miR-150 in atherosclerosis and the relation between miR-150 and PDLIM1 provided us with some potential evidence for the contribution of PDLIM1 in the inflammation. ${ }^{36}$ In this study, it was found that additional PDLIM1 treatment would lead to increased retinal leakage and inflammatory factors. Advanced experiments showed that miR-200a could relieve the retinal leakage and inflammation induced by PDLIM1 treatment. The data from in-vitro study showed that PDLIM1 was the target gene of miR-200a, and miR-200a demonstrated significant protective effects in both cell viability and apoptosis. However, no previous study focused on the role of PDLIM1 in the ECs. According to experiments on colorectal cancer cell lines, it was found that PDLIM1 could stabilize the E-cadherin/ $\beta$-catenin complex to inhibit the effect of $\beta$-catenin and 


\section{A}

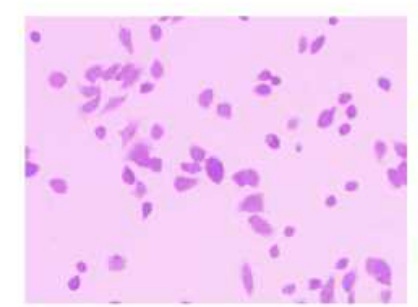

HG +miR-200a

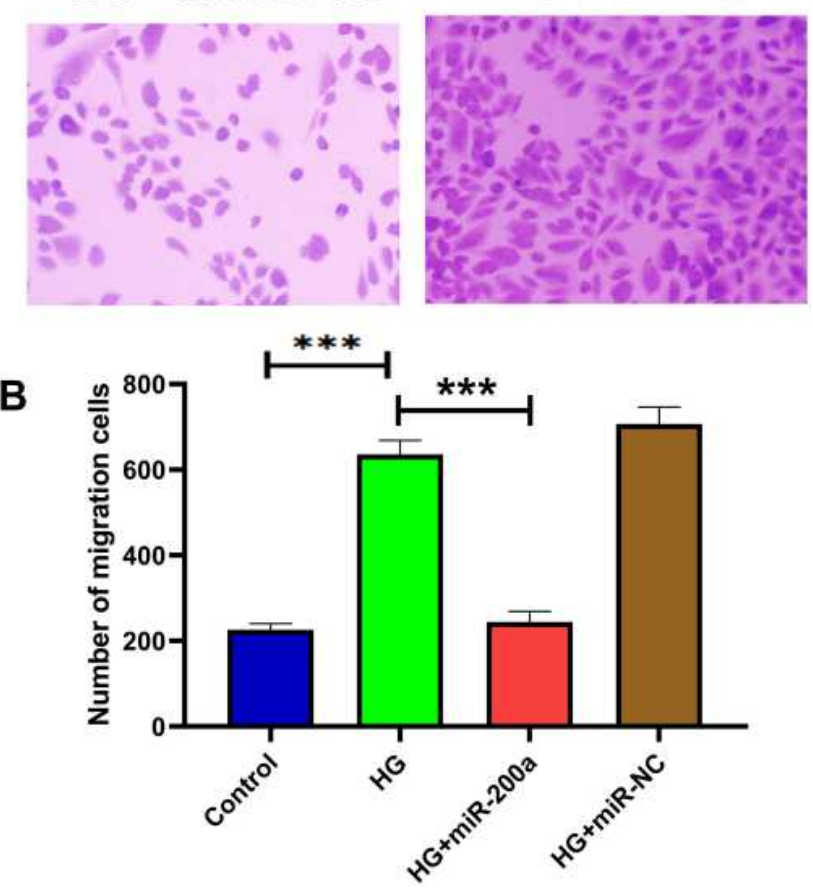

Figure 6 Effects of miR-200a on cell migration in high glucose (HG)-treated HRMECs. The migrated cell rate was detected in cultured HRMECs in (A) normal glucose (NG), high glucose (HG), HG+miR-200a and HG+miR-NC; (B) summary of apoptotic rates in different groups. HG treatment significantly increased apoptotic rate, while the apoptotic status was improved by miR-200a treatment. $* * * p<0.001$. $n=5$.

thus prevent epithelial-mesenchymal transition progress. As the E-cadherin/ $\beta$-catenin pathway has an important role in the blood-retina barrier (BRB) formation, ${ }^{37}$ PDLIM1 might have a pathetical role through Wnt pathway. However, only PDLIM1 mRNA expression was detected in current version, and this limited the understanding of the role of PDLIM1 in DR. In the following experiments, Western blot, cross-section and retinal flat mountain data of PDLIM1 would provide us with more knowledge.

miRNAs demonstrated their roles through negatively regulating gene expression post-transcriptionally by binding to target mRNAs for or inhibiting translation. According to previous studies, it has been reported that quaking $(\mathrm{QKI})$, bromodomain containing protein 4 (BRD4), protocadherin 9 (PDCH9) and others could work as the target genes of miR-200a. ${ }^{38-40}$ In this study, we confirmed that PDLIM1 was another target for miR200a as discussed above. As macular edema is associated with damaged vision activity and miR-200a might demonstrate protective effects in DR, we detected the retinal permeability through both retinal thickness by OCT and retinal leakage by Evans Blue assay. It was found that retinal thickness was not higher than the normal control, and we hypothesized that this was due to retinal thickening because BRB damage did not compete with retinal thinning due to cell loss in the STZ-induced DM mice animal model. Thus, we conducted advanced studies including PDLIM1 and PDLIM1 in combination with miR-200a treatment groups. It was found that miR200a could extenuate PDLIM1-induced retinal thickness increase partly. When the retinal leakage was considered, it was found that diabetic mice tend towards retinal leakage more than do control mice. After exogenous PDLIM1 was applied, retinal leakage was far more severe than that in the normal mice and was blocked by miR-200a injection completely. The data from in-vivo edema-related studies showed that PDLIM1 produced important effects in the BRB damage, and miR-200a was a potential therapy target for diabetes-related macular edema.

The clinical and experimental samples showed that retinal microangiopathy and neuronal function impairment appear in early-stage DM, and these lesions are closely related to apoptosis. ${ }^{41}$ Therefore, the role of retinal apoptosis in the mechanism of retinopathy and its regulation was studied and early-stage diabetes. Considering that the loss of function and structure of retinal microvascular ECs were related with microvascular nonperfusion, capillary network degradation and then induced a series of dysfunction. ${ }^{42}$ Interventions to block the development of DR have important clinical significance, and we tested the potential protective effect of miR-200a. Based on the data of flow cytometry as well as protein expression of apoptosis-related key genes, we concluded that miR-200a could produce remarkable anti-apoptotic effects through the mitochondrial pathway. The anti-apoptosis effect of miR-200a was reported in retinal ganglion cells ${ }^{43}$ and neuronal PC12 cells. ${ }^{44}$ The protective effect of miR-200a on different neural cells in different degenerative disorders suggest additional benefits other than the protection of ECs. 
A

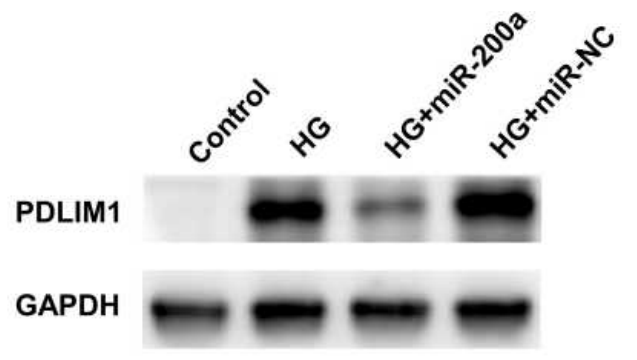

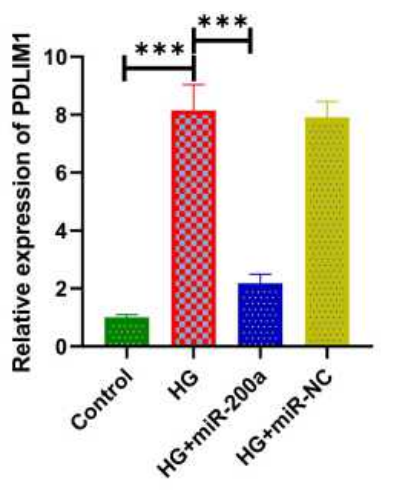

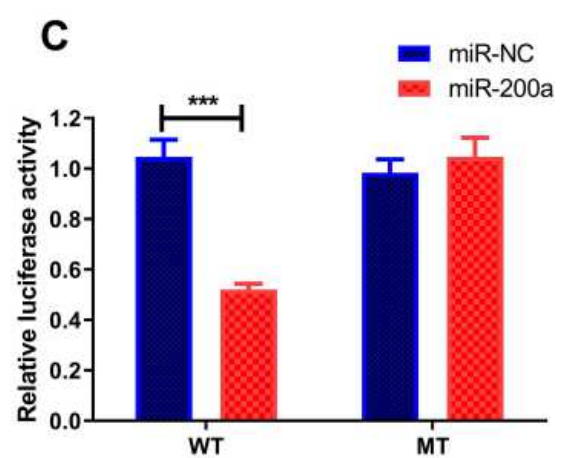

\section{B PDLIM13' UTR 5' $\quad$ '..UGCAGCUUUUUCUCUCAGUGUU C... hsa-miR-200a 3 ' UGUAGCAAUGGUCUGUCACAA U}

Figure 7 PDLIMI was a target gene of miR-200a. (A) Protein expression of PDLIMI was evaluated by Western blot; (B) miR-200a binds with the wild-type PDLIMI 3-UTR, cloned into the dual-luciferase vector; (C) the relative luciferase activity was determined in 293T cells transfected with miR-200a/miR-NC and recombinant dual-luciferase vector. $* * * P<0.001 . n=3$.

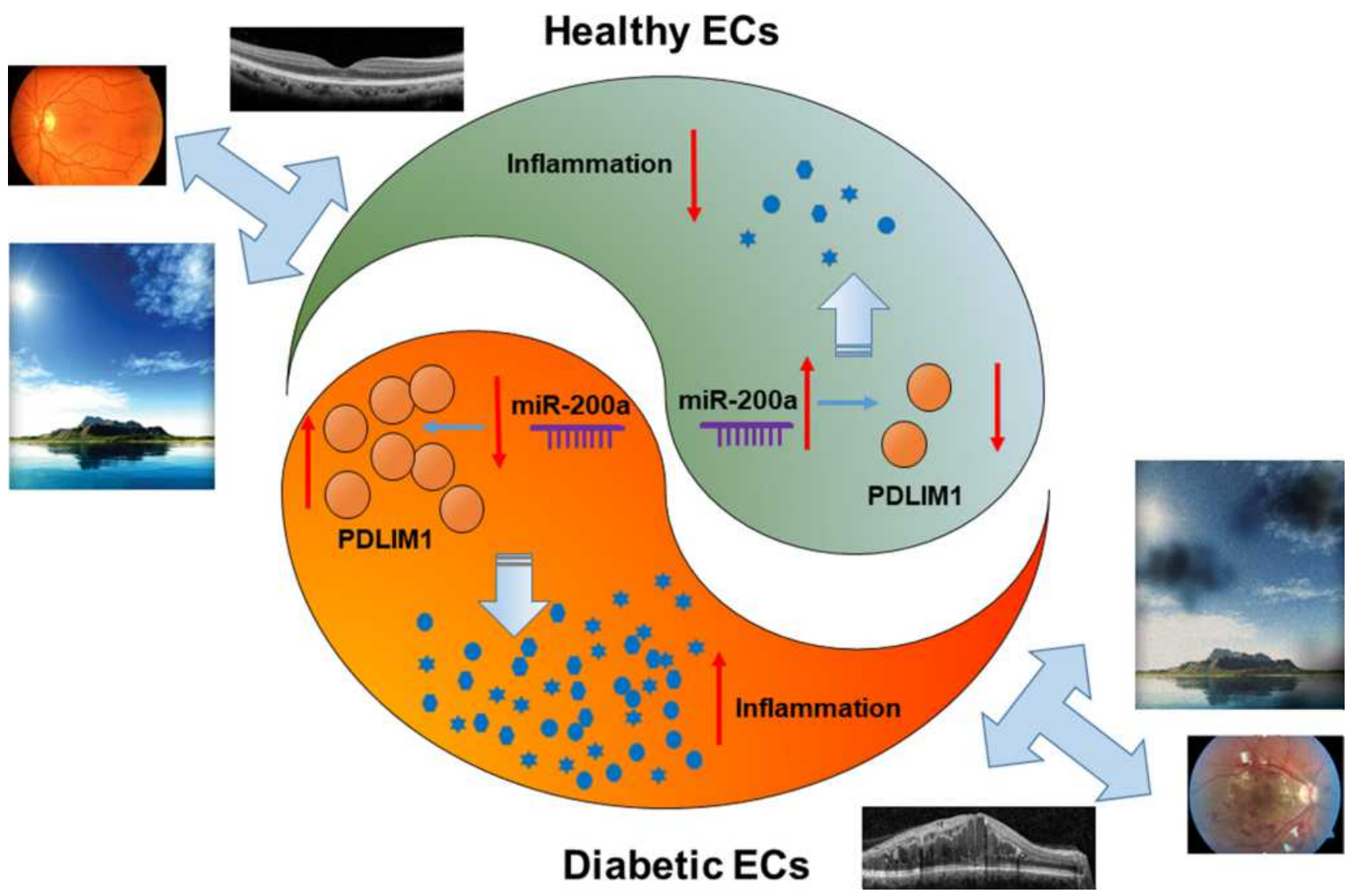

Figure 8 miR-200a protected DR through down-regulating PDLIMI expression. miR-200a can down-regulate PDLIMI in retinal vascular endothelial cells and thus decrease the expressions of inflammatory factors. As the retinal leakage and angiogenesis would be alleviated, there would be significant improvement in vision.

\section{Conclusions}

Collectively, these results based on both in-vivo and in-vitro data show that the miR-200a plays important roles in the development of DR that affect HRMECs viability, apoptosis and cell migration. As treatment with miR-200a could reduce retinal permeability and inhibit inflammation, we postulate that 
miR-200a could be a potential therapy target through downregulating PDLIM1 in DR.

\section{Acknowledgments}

This study was supported by Youth Project of Henan Provincial Health and Health Commission, Ministry of Education (SB201902008). We thank Dr Tao Peng of Zhengzhou University for assisting in study design.

\section{Author Contributions}

All authors made a significant contribution to the work reported, whether that is in the conception, study design, execution, acquisition of data, analysis and interpretation, or in all these areas; took part in drafting, revising or critically reviewing the article; gave final approval of the version to be published; have agreed on the journal to which the article has been submitted; and agree to be accountable for all aspects of the work.

\section{Disclosure}

The authors declare no conflicts of interest for this work.

\section{References}

1. Andes LJ, Li Y, Srinivasan M, Benoit SR, Gregg E, Rolka DB. Diabetes prevalence and incidence among medicare beneficiaries United States, 2001-2015. MMWR Morb Mortal Wkly Rep. 2019;68 (43):961-966. doi:10.15585/mmwr.mm6843a2

2. Zhu W, Meng YF, Wu Y, Xu M, Lu J. Association of alcohol intake with risk of diabetic retinopathy: a meta-analysis of observational studies. Sci Rep. 2017;7(1):4. doi:10.1038/s41598-017-00034-w

3. Marcovecchio ML, Dalton RN, Daneman D, et al., Adolescent type 1 Diabetes cardio-renal Intervention Trial study g. A new strategy for vascular complications in young people with type 1 diabetes mellitus. Nat Rev Endocrinol. 2019;15(7):429-435. doi:10.1038/s41574-0190198-2

4. Shi Y, Vanhoutte PM. Macro- and microvascular endothelial dysfunction in diabetes. $J$ Diabetes. 2017;9(5):434-449. doi:10.1111/17530407.12521

5. Wang N, Zhang C, Xu Y, et al. OMICs approaches-assisted identification of macrophages-derived MIP-1gamma as the therapeutic target of botanical products TNTL in diabetic retinopathy. Cell Commun Signal. 2019;17(1):81. doi:10.1186/s12964-019-0396-5

6. Capitao M, Soares R. Angiogenesis and inflammation crosstalk in diabetic retinopathy. J Cell Biochem. 2016;117(11):2443-2453. doi: $10.1002 /$ jcb. 25575

7. Scholz A, Plate KH, Reiss Y. Angiopoietin-2: a multifaceted cytokine that functions in both angiogenesis and inflammation. Ann N Y Acad Sci. 2015;1347:45-51. doi:10.1111/nyas. 12726

8. Wong TY, Cheung CM, Larsen M, Sharma S, Simo R. Diabetic retinopathy. Nat Rev Dis Primers. 2016;2:16012. doi:10.1038/ nrdp.2016.12

9. Sodhi A, Ma T, Menon D, et al. Angiopoietin-like 4 binds neuropilins and cooperates with VEGF to induce diabetic macular edema. J Clin Invest. 2019;129(11):4593-4608. doi:10.1172/JCI120879

10. Wang W, Lo ACY. Diabetic retinopathy: pathophysiology and treatments. Int J Mol Sci. 2018;19(6).
11. Mehta H, Hennings C, Gillies MC, Nguyen V, Campain A, FraserBell S. Anti-vascular endothelial growth factor combined with intravitreal steroids for diabetic macular oedema. Cochrane Database Syst Rev. 2018;4:CD011599. doi:10.1002/14651858.CD011599.pub2

12. Yokomizo H, Maeda Y, Park K, et al. Retinol binding protein 3 is increased in the retina of patients with diabetes resistant to diabetic retinopathy. Sci Transl Med. 2019;11:499. doi:10.1126/scitranslmed. aau6627

13. Lin JB, Moolani HV, Sene A, et al. Macrophage microRNA-150 promotes pathological angiogenesis as seen in age-related macular degeneration. JCI Insight. 2018;3(7). doi:10.1172/jci.insight.120157

14. Ren C, Liu Q, Wei Q, et al. Circulating miRNAs as potential biomarkers of age-related macular degeneration. Cell Physiol Biochem. 2017;41(4):1413-1423. doi:10.1159/000467941

15. Shafabakhsh R, Aghadavod E, Mobini M, Heidari-Soureshjani R, Asemi Z. Association between microRNAs expression and signaling pathways of inflammatory markers in diabetic retinopathy. $J$ Cell Physiol. 2019;234(6):7781-7787. doi:10.1002/jcp.27685

16. Biswas S, Sarabusky M, Chakrabarti S. Diabetic retinopathy, IncRNAs, and inflammation: a dynamic, interconnected network. J Clin Med. 2019;8(7):1033. doi:10.3390/jcm8071033

17. Zhang J, Cui C, Xu H. Downregulation of miR-145-5p elevates retinal ganglion cell survival to delay diabetic retinopathy progress by targeting FGF5. Biosci Biotechnol Biochem. 2019;83 (9):1655-1662. doi:10.1080/09168451.2019.1630251

18. Dong N, Wang Y. MiR-30a regulates S100A12-induced retinal microglial activation and inflammation by targeting NLRP3. Curr Eye Res. 2019;44(11):1236-1243. doi:10.1080/02713683.2019.1632350

19. Gong Q, Xie J, Li Y, Liu Y, Su G. Enhanced ROBO4 is mediated by up-regulation of HIF-1alpha/SP1 or reduction in miR-125b-5p/miR146a-5p in diabetic retinopathy. $J$ Cell Mol Med. 2019;23 (7):4723-4737. doi:10.1111/jcmm.14369

20. Ding Y, Hu Z, Luan J. Protective effect of miR-200b/c by inhibiting vasohibin-2 in human retinal microvascular endothelial cells. Life Sci. 2017;191:245-252. doi:10.1016/j.lfs.2017.09.001

21. You ZP, Zhang YL, Shi K, Shi L, Zhang YZ. Suppression of diabetic retinopathy with GLUT1 siRNA. Sci Rep. 2017;7(1):7437. doi:10.1038/s41598-017-07942-x

22. Zhu K, Hu X, Chen H, et al. Downregulation of circRNA DMNT3B contributes to diabetic retinal vascular dysfunction through targeting miR-20b-5p and BAMBI. EBioMedicine. 2019;49:341-353. doi:10.1016/j.ebiom.2019.10.004

23. Blindbaek SL, Peto T, Grauslund J. How do we evaluate the role of focal/grid photocoagulation in the treatment of diabetic macular edema? Acta Ophthalmol. 2019;97(4):339-346. doi:10.1111/aos.13997

24. Babiuch AS, Han M, Conti FF, Wai K. Association of disorganization of retinal inner layers with visual acuity response to anti-vascular endothelial growth factor therapy for macular edema secondary to retinal vein occlusion. JAMA Ophthalmol. 2019;137(1):38-46. doi:10.1001/jamaophthalmol.2018.4484

25. Namba R, Kaneko H, Suzumura A, et al. In vitro epiretinal membrane model and antibody permeability: relationship with Anti-VEGF resistance in diabetic macular edema. Invest Ophthalmol Vis Sci. 2019;60(8):2942-2949. doi:10.1167/iovs.19-26788

26. Rubsam A, Parikh S, Fort PE. Role of inflammation in diabetic retinopathy. Int J Mol Sci. 2018;19(4):942. doi:10.3390/ ijms19040942

27. Zhu W, Wu Y, Meng YF, Xing Q, Tao JJ, Lu J. Association of obesity and risk of diabetic retinopathy in diabetes patients: a meta-analysis of prospective cohort studies. Medicine (Baltimore). 2018;97(32): e11807. doi:10.1097/MD.0000000000011807

28. Eshaq RS, Wright WS, Harris NR. Oxygen delivery, consumption, and conversion to reactive oxygen species in experimental models of diabetic retinopathy. Redox Biol. 2014;2:661-666. doi:10.1016/j. redox.2014.04.006 
29. Zhu W, Meng YF, Xing Q, Tao JJ. Identification of IncRNAs involved in biological regulation in early age-related macular degeneration. Int $J$ Nanomedicine. 2017;12:7589-7602. doi:10.2147/IJN.S140275

30. Panda H, Pelakh L, Chuang TD, Luo X. Endometrial miR-200c is altered during transformation into cancerous states and targets the expression of ZEBs, VEGFA, FLT1, IKKbeta, KLF9, and FBLN5. Reprod Sci. 2012;19(8):786-796. doi:10.1177/1933719112438448

31. Wu H, Kong L, Tan Y, et al. C66 ameliorates diabetic nephropathy in mice by both upregulating NRF2 function via increase in miR-200a and inhibiting miR-21. Diabetologia. 2016;59(7):1558-1568. doi:10.1007/s00125-016-3958-8

32. Sharma P, Gupta S, Chaudhary M, et al. Oct4 mediates Muller glia reprogramming and cell cycle exit during retina regeneration in zebrafish. Life Sci Alliance. 2019;2(5):e201900548. doi:10.26508/ lsa.201900548

33. Bauer K, Kratzer M, Otte M, et al. Human CLP36, a PDZ-domain and LIM-domain protein, binds to alpha-actinin-1 and associates with actin filaments and stress fibers in activated platelets and endothelial cells. Blood. 2000;96(13):4236-4245. doi:10.1182/blood. V96.13.4236

34. Huang Z, Zhou JK, Wang K, et al. PDLIM1 inhibits tumor metastasis through activating Hippo signaling in hepatocellular carcinoma. Hepatology. 2019.

35. Shang Y, Wang H, Jia P, et al. Autophagy regulates spermatid differentiation via degradation of PDLIM1. Autophagy. 2016;12 (9):1575-1592. doi:10.1080/15548627.2016.1192750

36. Gong FH, Cheng WL, Wang H, et al. Reduced atherosclerosis lesion size, inflammatory response in miR-150 knockout mice via macrophage effects. J Lipid Res. 2018;59(4):658-669. doi:10.1194/jlr. M082651
37. Zhang C, Lai MB, Pedler MG, Johnson V, Adams RH, Petrash JM. Endothelial cell-specific inactivation of TSPAN12 (Tetraspanin 12) reveals pathological consequences of barrier defects in an otherwise intact vasculature. Arterioscler Thromb Vasc Biol. 2018;38 (11):2691-2705. doi:10.1161/ATVBAHA.118.311689

38. Kim EJ, Kim JS, Lee S, et al. QKI, a miR-200 target gene, suppresses epithelial-to-mesenchymal transition and tumor growth. Int J Cancer. 2019;145(6):1585-1595. doi:10.1002/ijc.32372

39. Guan H, You Z, Wang C, et al. MicroRNA-200a suppresses prostate cancer progression through BRD4/AR signaling pathway. Cancer Med. 2019;8(4):1474-1485. doi:10.1002/cam4.2029

40. Shi C, Yang Y, Zhang L, et al. MiR-200a-3p promoted the malignant behaviors of ovarian cancer cells through regulating PCDH9. Onco Targets Ther. 2019;12:8329-8338. doi:10.2147/OTT.S220339

41. Al-Kharashi AS. Role of oxidative stress, inflammation, hypoxia and angiogenesis in the development of diabetic retinopathy. Saudi J Ophthalmol. 2018;32(4):318-323. doi:10.1016/j.sjopt.2018.05.002

42. Whitehead M, Wickremasinghe S, Osborne A. Diabetic retinopathy: a complex pathophysiology requiring novel therapeutic strategies. Expert Opin Biol Ther. 2018;18(12):1257-1270. doi:10.1080/ 14712598.2018.1545836

43. Peng H, Sun YB, Hao JL, Lu CW. Neuroprotective effects of overexpressed microRNA-200a on activation of glaucoma-related retinal glial cells and apoptosis of ganglion cells via downregulating FGF7-mediated MAPK signaling pathway. Cell Signal. 2019;54:179-190. doi:10.1016/j.cellsig.2018.11.006

44. Zhang QS, Liu W, Lu GX. miR-200a-3p promotes b-Amyloidinduced neuronal apoptosis through down-regulation of SIRT1 in Alzheimer's disease. J Biosci. 2017;42(3):397-404. doi:10.1007/ s12038-017-9698-1
Journal of Inflammation Research

\section{Publish your work in this journal}

The Journal of Inflammation Research is an international, peerreviewed open-access journal that welcomes laboratory and clinical findings on the molecular basis, cell biology and pharmacology of inflammation including original research, reviews, symposium reports, hypothesis formation and commentaries on: acute/chronic inflammation; mediators of inflammation; cellular processes; molecular

\section{Dovepress}

mechanisms; pharmacology and novel anti-inflammatory drugs; clinical conditions involving inflammation. The manuscript management system is completely online and includes a very quick and fair peerreview system. Visit http://www.dovepress.com/testimonials.php to read real quotes from published authors. 